\title{
Relaciones entre densidades y biomasas de Salmo trutta fario y mediciones del mesohábitat en tramos trucheros de la Comunidad Valenciana
}

\author{
Juan Diego Alcaraz-Hernández, Francisco Martínez-Capel*, Matías Peredo, \\ Aina Berta Hernández-Mascarell
}

Departamento de Ingeniería Hidráulica y Medio Ambiente. Escuela Politécnica Superior de Gandía. Ctra. Nazaret-Oliva s/n, Gandía 46730, Valencia, España.

* Autor responsable de la correspondencia: fmcapel@dihma.upv.es

\section{RESUMEN}

Relaciones entre densidades y biomasas de Salmo trutta fario y mediciones del mesohábitat en tramos trucheros de la Comunidad Valenciana

En el año 2003 se estudiaron las poblaciones autóctonas de Salmo trutta fario en los ríos valencianos. Por otro lado se midieron las condiciones del hábitat según las sucesivas unidades hidro-morfológicas en tramos de 300 m de longitud, junto con variables geográficas y de calidad del agua. Con el objeto de relacionar densidades y biomasas con el hábitat, todas las variables independientes fueron introducidas en modelos de regresión múltiple (para la densidad y la biomasa relativa de trucha común). Mediante análisis paso a paso eliminatorio quedaron seleccionadas las más significativas para cada uno de los dos modelos multivariantes, obteniéndose en todos un bajo coeficiente de correlación pero con una significación adecuada $(p<0.10)$. Las variables establecidas en los dos modelos fueron la anchura del cauce (con signo negativo) y el porcentaje de cantos rodados (64-256 mm) en cada mesohábitat. La superficie, así como la profundidad media y máxima no aparecieron en el modelo final, pero se demostró su correlación con la anchura del cauce. Todas estas variables estaban englobadas dentro del grupo de características del mesohábitat, habiéndose descartado las demás que no fueron significativas a la escala del estudio.

Palabras clave: Salmo trutta, densidad, biomasa, mesohábitat, unidades hidro-morfológicas.

\begin{abstract}
Relationships between density and biomass of Salmo trutta fario and mesohabitat variables in trout streams of the Valencian region

In the year 2003, populations of Salmo trutta fario were studied in the Valencian rivers. Habitat conditions were also studied along the successive hydro-morphological units in $300 \mathrm{~m}$ long reaches, along with geographic and water-quality variables. With the objective of relating density and biomass with the habitat, all the independent variables were used in a stepwise multiple regression analysis (for common trout density and relative biomass). Through a step-wise analysis, the most significant variables were chosen for each of the two multivariate models, showing all a low correlation coefficient, but with good significance $(\mathrm{p}<0.10)$. The most relevant variables in both models were stream width (negative correlation) and percentage of cobbles $(64-256 \mathrm{~mm})$ in each mesohabitat. The water surface area, and mean and maximum depth did not appear in the final model, but their correlation with stream width was demonstrated. All these variables were included within the mesohabitat characteristics, having being discarded the rest that were not significant at the scale of the study.
\end{abstract}

Keywords: Salmo trutta, density, biomass, mesohabitat, hydro-morphological units. 


\section{INTRODUCCIÓN}

En la Comunidad Valenciana, las poblaciones de trucha común, Salmo trutta fario L., se localizan en ríos de cabecera, que descienden desde las sierras de orientación NE-SO hacia la vertiente mediterránea. Estas poblaciones son autóctonas y están incluidas dentro del linaje genético del mediterráneo (Machordom et al., 2000). En las últimas décadas el área de distribución de la especie en los ríos valencianos ha disminuido de forma drástica, por lo que la Consejería de Territorio y Vivienda de la Comunidad Valenciana decidió gestionar estas poblaciones para su conservación. La causa de esta disminución se debe, en parte, a la gran actividad humana que altera los ecosistemas fluviales (Boon et al., 1992; González del Tánago \& García de Jalón, 2001). Las perturbaciones observadas son, entre otras, la fuerte regulación de los caudales, la destrucción del hábitat, la contaminación de las aguas y la introducción de especies exóticas (Almodóvar et al., 2004).

El objetivo de este estudio es identificar, a escala de unidades hidro-morfológicas, el grupo de variables más significativas (entre las que cumplen normalidad) que determinan las variaciones de su densidad y biomasa. Para ello se han construido dos modelos, uno de densidad y otro de biomasa de trucha común. A medio-largo plazo, la finalidad de nuestro estudio es aportar datos que sirvan de herramienta en futuras restauraciones, mejoras y gestión de los ríos trucheros.

\section{ÁREA DE ESTUDIO}

El presente trabajo se ha desarrollado en cuatro ríos de montaña, a una altura entre los 575 y $951 \mathrm{~m}$ sobre el nivel del mar, todos ellos están ubicados en la Comunidad Valenciana (Fig. 1). En ellos se han seleccionado 20 puntos (Tabla 1) donde se dan las condiciones necesarias para el desarrollo de poblaciones naturales autosostenidas de la trucha común autóctona, cohabitando en algunos puntos con proporciones variables de trucha arco iris. Los muestreos fueron realizados en los meses de junio y julio de 2003. El único río donde no se capturó trucha arco iris fue el Villahermosa. En este estudio no se han incluido otras especies capturadas como Anguilla anguilla (L.), Barbus guiraonis (Steind.), Barbus haasi (Mertens) Chondrostoma turiense (Elvira), Chondrostoma arcasii (Steind.) y Squalius pyrenaicus (Günther).

Según la clasificación de Strahler (1957), el orden fluvial en los ríos Ebrón y Vallanca es 2 y en los ríos Palancia y Villahermosa es 3. Los ríos Ebrón y Vallanca desembocan en el Turia. El Ebrón tiene $41 \mathrm{~km}$ de longitud y el Vallanca $20 \mathrm{~km}$, dándose las condiciones necesarias en la totalidad de ambos ríos para albergar poblaciones estables de trucha común. La anchura máxima que se midió en el río Ebrón fue de 6.90 m y en el Vallanca de $5.67 \mathrm{~m}$. El río Palancia desemboca directamente en el mar Mediterráneo con una longitud aproximada de $92 \mathrm{~km}$, pero sólo los primeros $40 \mathrm{~km}$ cuentan con poblaciones estables de trucha. La anchura máxima medida en los

Tabla 1. Localización de cada tramo mediante las coordenadas U.T.M. del punto central de los $300 \mathrm{~m}$ recorridos. Location of each stretch by means of the U.T.M. coordinates corresponding to the central point of the $300 \mathrm{~m}$ studied.

\begin{tabular}{lcc}
\hline Río & X-UTM & Y-UTM \\
\hline Ebrón & 647676.52 & 4439918.04 \\
Ebrón & 647578.65 & 4439970.74 \\
Ebrón & 647483.29 & 4439988.32 \\
Ebrón & 647355.30 & 4440101.24 \\
Ebrón & 646001.58 & 4441217.62 \\
Ebrón & 643707.29 & 4444988.70 \\
Ebrón & 643733.66 & 4445102.97 \\
Vallanca & 646456.79 & 4436006.47 \\
Vallanca & 646415.72 & 4435924.32 \\
Vallanca & 645866.35 & 4435790.83 \\
Vallanca & 645825.27 & 4435770.29 \\
Vallanca & 645060.26 & 4435713.81 \\
Vallanca & 645003.78 & 4435703.55 \\
Vallanca & 641276.26 & 4436052.68 \\
Vallanca & 641260.86 & 4436088.62 \\
Palancia & 697604.40 & 4419501.94 \\
Palancia & 694877.41 & 4421466.87 \\
Villahermosa & 722803.37 & 4449028.50 \\
Villahermosa & 722653.77 & 4449681.30 \\
Villahermosa & 718260.97 & 4455121.30 \\
\hline
\end{tabular}




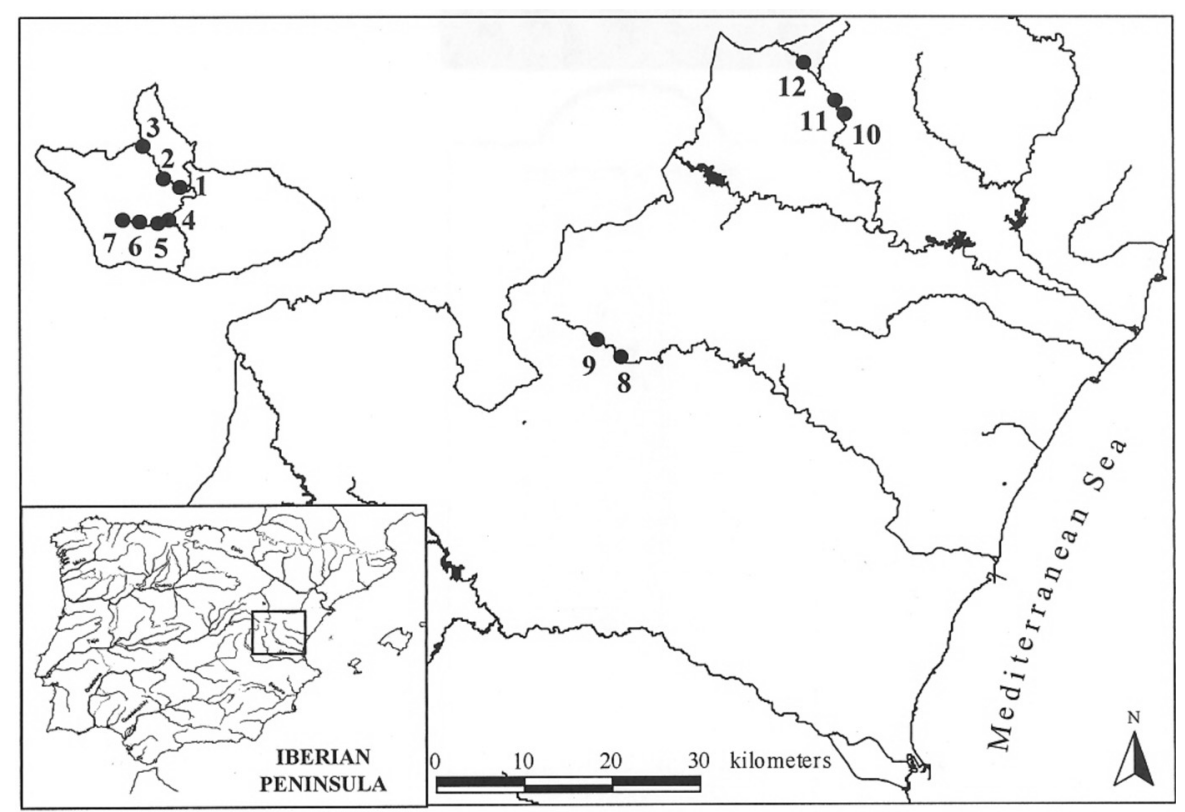

Figura 1. Mapa de localización en la Península Ibérica, con ampliación de la zona de ríos donde se realizaron las capturas en la Comunidad Valenciana. Río Ebrón $(1,2,3)$, río Vallanca $(4,5,6,7)$, río Palancia $(8,9)$ y río Villahermosa $(10,11,12)$. Map of the Iberian Peninsula showing the study region (down left). The sampling sites are shown in the main view, in the four streams Ebrón (1, 2, 3), Vallanca (4, 5, 6, 7), Palancia (8, 9) and Villahermosa $(10,11,12)$.

tramos estudiados fue de $9.65 \mathrm{~m}$. Por último, el río Villahermosa vierte sus aguas en el Mijares teniendo una longitud de $67 \mathrm{~km}$, de los cuales los $33 \mathrm{~km}$ iniciales son aptos para la trucha, con una anchura máxima medida de $13.5 \mathrm{~m}$. Las pendientes son elevadas por tratarse de ríos de cabecera, situándose la media de todos ellos en el intervalo de $21.8 \mathrm{~m} / \mathrm{km} \pm$ S.D. 4.8. La cuenca más pequeña es de de $55 \mathrm{~km}^{2}$ en el tramo de estudio más alto del río Vallanca y la mayor es de $250 \mathrm{~km}^{2}$ en el tramo bajo del río Ebrón, siendo la media de todos los puntos de muestreo de $175 \mathrm{~km}^{2} \pm$ S.D. 81.5 .

En las cuatro cuencas, el porcentaje del uso del suelo forestal esta entre el 77 y $95 \%$, siendo la media de $84 \% \pm$ S.D. 5.9. El resto del porcentaje de uso del suelo abarca las superficies urbanas y las zonas agrícolas. Las cuencas están formadas por rocas carbonatadas, abarcando un porcentaje medio del $96 \% \pm$ S.D. 4.1. Esta naturaleza geológica influye directamente en la naturaleza fisicoquímica de sus aguas. La dureza media del agua durante los dos meses de trabajo fue de $291.8 \mathrm{mg} / 1$ de $\mathrm{CaCO}_{3} \pm$ S.D. 29.6. Esta dureza le da un carácter básico al agua, con un $\mathrm{pH}$ medio de $7.9 \pm$ S.D. 0.2. La conductividad media del agua fue de $55.5 \mu \mathrm{S} / \mathrm{cm} \pm$ S.D. 8.0. $\mathrm{La}$ temperatura media de muestreo en los cuatro ríos fue $15.7^{\circ} \mathrm{C} \pm$ S.D. 1.8. La precipitación media anual varía entre $442 \mathrm{~mm}$ en el río Ebrón, 455 $\mathrm{mm}$ en el río Vallanca, $571 \mathrm{~mm}$ en el río Palancia y $583 \mathrm{~mm}$ en el río Villahermosa. En general la calidad del agua en todos los tramos es muy buena y los vertidos orgánicos de poblaciones no son significativos a escala global, a juzgar por los análisis estivales realizados hasta el momento.

\section{METODOLOGÍA}

\section{Toma de datos}

Los muestreos se realizaron por el día, durante los meses de Junio y Julio de 2003, cuando los caudales circulantes del río eran bajos (aunque no mínimos). Se capturaron un total de 224 truchas comunes y 53 arco iris mediante pesca eléctrica, mediante capturas sucesivas sin 
reemplazamiento. Para calcular las densidades (ind./ha) y biomasas ( $\mathrm{kg} / \mathrm{ha}$ ) se utilizó el método de máxima verosimilitud ponderada de Carle \& Strub (1978), por ser el de mayor robustez estadística (Cowx, 1983).

Las unidades de muestreo fueron los mesohábitats o unidades hidro-morfológicas. En total se muestrearon 20 tramos de $300 \mathrm{~m}$, centrados en los puntos de captura de peces por pesca eléctrica. Esta longitud se estableció considerando estudios de Leopold et al., (1964), que estimaron que el espaciamiento natural de rápidos y remansos de alrededor de 6 veces la anchura del cauce, y otras recomendaciones que cifran entre 150 y 300 metros la longitud más aconsejable (Meador et al., 1993).

La tipificación y caracterización del mesohábitat se realizó siguiendo los criterios para ríos de salmónidos establecidos para la técnica Basinwide Visual Estimation Technique, o BVET (Dolloff et al., 1993). Esta metodología fue simplificada para separar las unidades hidromorfológicas en 2 tipos; (1) lentos que englobarían a los lentos (profundidades mayores de 0.6 $\mathrm{m}$ y velocidades inferiores a la media del tramo) y las tablas (profundidades menores de $0.6 \mathrm{~m}$, velocidades similares a la media el tramo y sin turbulencias apreciables), (2) rápidos que incluirían a las corrientes (aguas poco profundas con turbulencias superficiales y velocidades del agua inferiores a $0.4 \mathrm{~m} / \mathrm{s}$ ) y a los rápidos (aguas someras con velocidades mayores que la media del tramo, con abundantes turbulencias superficiales, elementos de sustrato que sobresalen del agua y predominio del flujo supercrítico).

En cada mesohábitat se registraron las siguientes variables: longitud del mesohábitat (m), anchura media (m) del agua tomada como media de tres puntos que corresponden a 1/4, 1/2, y 3/4 de la longitud total de la unidad, profundidad media (m) tomada como media de nueve puntos correspondientes a la medida tomada en $1 / 4,1 / 2$, y $3 / 4$ en cada transecto donde se midió la anchura, profundidad máxima $(\mathrm{m})$ y porcentaje de sustrato (\%) estimado visualmente. Los tipos de sustrato (según la clasificación de la American Geophysical Union simplificada) considerados fueron: roca madre (roca continua), grandes bloques (> $1.024 \mathrm{~mm}$ de diámetro), bolos (256$1.024 \mathrm{~mm})$, cantos $(64-256 \mathrm{~mm})$, gravas $(8-64$ $\mathrm{mm})$, gravillas $(2-8 \mathrm{~mm})$, arenas $(62 \mu \mathrm{m}-2 \mathrm{~mm})$, limos $(4-62 \mu \mathrm{m}) \mathrm{y}$ arcillas $(<4 \mu \mathrm{m})$.

Una vez estudiado cada tramo de $300 \mathrm{~m}$, se calcularon las variables independientes que se han introducido en los análisis estadísticos, y que se asocian a cada punto de muestreo. Desde el punto de vista del mesohábitat, estas fueron la superficie $\left(\mathrm{m}^{2}\right)$, el porcentaje de cantos rodados y también el de gravas en cada mesohábitat y la representatividad de hábitats lentos en el tramo correspondiente (porcentaje longitud en $300 \mathrm{~m}$ ). Las variables geográficas consideradas fueron la altitud del punto de estudio ( $\mathrm{m}$ ), pendiente $(\mathrm{m} / \mathrm{km})$, distancia hasta el nacimiento $(\mathrm{km})$ y distancia hasta la desembocadura $(\mathrm{km})$. Dichas variables fueron estimadas a partir de la cartografía digital escala 1:10 000, proporcionada por el Instituto Cartográfico Valenciano. En cuanto a la calidad del agua, se recogieron tres muestras a lo largo de cada río, para medir la dureza del agua. Ésta se estimó utilizando métodos estandarizados descritos según la APHA (1998). Otras variables citadas en la literatura científica que influyen en las poblaciones de trucha, como puede ser el caudal (Lobón-Cerviá \& Mortensen, 2005) no se ha podido medir porque solamente se contaba con una estación de aforo, en el río Ebrón.

\section{Análisis estadístico}

En primer lugar, se analizaron los datos de cada variable para comprobar los supuestos subyacentes a la regresión simple y múltiple, como son la linealidad, normalidad y homocedasticidad de los datos. Para confirmar la normalidad de las variables se aplicaron los test de normalidad de chi-cuadrado y de bondad de ajuste de Kolmogorov-Smirnov $(p<0.10)$. Puesto que la mayoría de ellas no cumplían con la normalidad, se probaron distintas transformaciones con funciones sencillas. La mejor transformación resultó ser la raíz cuadrada, aunque también se ha empleado la inversa y el logaritmo de la variable según el caso. El criterio seguido en la elección de la función de transformación fue que las variables transformadas cumplieran con las dos pruebas 
de normalidad realizadas. La homogeneidad de las varianzas se comprobó aplicando la prueba de Levene $(p<0.10)$.

Con las variables transformadas se calcularon las regresiones lineales por pares, entre variables independientes, y también entre cada variable independiente y las dos dependientes (densidad y biomasa) y se realizó un análisis de las varianzas (ANOVA) en cada caso. Basándose en esta prueba estadística, se descartaron de los análisis las variables independientes que no presentaban una relación significativa $(p \geq 0.10)$ con ninguna de las variables dependientes. Con el resto de las variables se ha aplicado un análisis de regresión múltiple mediante eliminación regresiva de las variables independientes hasta obtener los modelos finales de densidad y biomasa.

\section{RESULTADOS}

En primer lugar, el proceso de exclusión de variables, por falta de normalidad, afectó a la conductividad del agua, pendiente del tramo, distancia hasta el nacimiento y distancia hasta la desembocadura y longitud (Tabla 2), las demás variables fueron transformadas para cumplir con el principio de normalidad, excepto la representatividad de lentos que ya presentaban una distribución normal (además, todas las variables normales han cumplido con la premisa de homocedasticidad y linealidad). En la Tabla 2 se muestran las variables consideradas en el estudio, especificando las transformaciones utilizadas para alcanzar la normalidad.

Posteriormente, el análisis de la varianza mostró que algunas variables no resultaban significativas para la densidad ni la biomasa por lo que no pudieron integrarse en ninguno de los dos modelos. En la Tabla 3 se muestran los resultados de este análisis directo entre cada variable dependiente e independiente, previa a la elaboración del modelo de regresión múltiple. Este fue el caso de la profundidad media, el porcentaje de gravas y la representatividad de lentos. La profundidad máxima fue excluida solo en el análisis de biomasa.

Considerando únicamente las variables significativas para la densidad y biomasa, la tabla de correlaciones entre variables permitió observar

Tabla 2. Variables, tamaño muestral $(\mathrm{N})$ y función de transformación de las variables para la obtención de la normalidad de los datos $(p<0.10)$. Variables, sample size $(N)$ and variable transformation functions used to approach data normality $(p<0.10)$.

\begin{tabular}{lllc}
\hline Variables & & $\mathrm{N}$ & Transformación \\
\hline Dependientes & Densidad de trucha común & & $\sqrt{x}$ \\
& Biomasa de trucha común & 20 & $\sqrt{x}$ \\
Independientes & & 20 & \\
- Características del agua & Conductividad de agua & & $\mathrm{NN}^{*}$ \\
- Características geográficas & & 20 & $\mathrm{NN}$ \\
& Altitud & 20 & $\mathrm{NN}$ \\
& Pendiente del tramo & 20 & $\mathrm{NN}$ \\
& Distancia hasta el nacimiento & 20 & $\mathrm{NN}$ \\
- Características del mesohábitatastancia hasta la desembocadura & Longitud & 20 & $\mathrm{NN}$ \\
& Anchura & 20 & $\sqrt{x}$ \\
& Superficie & 20 & $\log _{10} x$ \\
& Profundidad media & 20 & $\sqrt{x}$ \\
& Profundidad máxima & 20 & $\log _{10} x$ \\
& Cantos rodados & 20 & $\sqrt{x}$ \\
& Gravas & 20 & $\sqrt{x}$ \\
& Representatividad de lentos & 20 & - \\
\hline
\end{tabular}

${ }^{*} \mathrm{NN}$ : variable que no cumple la normalidad de los datos. 
Tabla 3. Significación de cada variable (transformadas) en el análisis ANOVA respecto a cada variable dependiente (D: Densidad, B: Biomasa). Significance of each variable (transformed) in the ANOVA analysis, regarding each dependent variable (D: Density, B: Biomass).

\begin{tabular}{lcc}
\hline & $\begin{array}{c}\text { D. de trucha } \\
\text { común }[\sqrt{x}]\end{array}$ & $\begin{array}{c}\text { B. de trucha } \\
\text { común }[\sqrt{x}]\end{array}$ \\
\hline Anchura $[\sqrt{x}]$ & 0.008 & 0.049 \\
Superficie $\left[\log _{10} x\right]$ & 0.055 & 0.094 \\
Prof. Media $[\sqrt{x}]$ & 0.235 & 0.904 \\
Prof. Máxima $\left[\log _{10} x\right]$ & 0.005 & 0.120 \\
Cantos Rodados $[\sqrt{x}]$ & 0.078 & 0.029 \\
Gravas & 0.499 & 0.547 \\
Representatividad Lentos & 0.169 & 0.176 \\
\hline
\end{tabular}

que existe una correlación lineal entre la profundidad máxima y tres variables: la anchura, la superficie y la profundidad media del mesohábitat. También existe dicha correlación, como es obvio, entre la anchura y la superficie.

Finalmente, se han obtenido mediante análisis de regresión múltiple dos modelos, uno de densidad y otro de biomasa, con un nivel de confianza superior o igual al $98 \%(p \leq 0.020)$. El modelo para la densidad de trucha común es significativo $(p<0.010)$ y las variables independientes del modelo explican el $45 \%$ de la va- riación observada (Tabla 4). Así pues la densidad es significativamente dependiente de la anchura (en sentido negativo) y del porcentaje de cantos rodados en el mesohábitat. De la misma manera el modelo para biomasa de trucha común es significativo $(p<0.020)$ y las variables independientes del modelo explican el $40 \%$ de la variación observada. El modelo de biomasa de trucha común es significativamente dependiente de la anchura (con signo negativo) y de los cantos rodados del mesohábitat (como con las densidades). Las restantes variables fueron excluidas en el proceso de eliminación paso a paso.

\section{DISCUSIÓN}

La primera observación a comentar es que las precisiones obtenidas en cada uno de los modelos han sido bajas (con $r^{2}$ no superiores al $50 \%$ ), por lo que no se podrán utilizar para inferir resultados. Sin embargo los modelos hallados son altamente significativos $(p<0.02)$, por lo que nos han servido para determinar algunas de las variables más influyentes en densidad y biomasa de trucha común. Además de estos modelos, podrían obtenerse otros que contuvieran otras variables, con coeficientes de correlación más elevados,

Tabla 4. Coeficiente de regresión de Pearson entre las variables independientes consideradas que resultaron significativas para la densidad y biomasa de trucha común (Prof.: profundidad; Rep. lentos: Representatividad de hábitats lentos). Se indica con dos asteriscos los casos en que $p<0.01$. Pearson's regression coefficient between independent variables that were significant for the brown trout density and biomass (Prof.: depth; Rep. lentos: Representativeness of slow habitats); two asterisks indicate $p<0.01$.

\begin{tabular}{|c|c|c|c|c|c|c|c|}
\hline & $\begin{array}{c}\text { Anchura } \\
{[\sqrt{x}]}\end{array}$ & $\begin{array}{c}\text { Superficie } \\
{\left[\log _{10} x\right]}\end{array}$ & $\begin{array}{l}\text { Prof. media } \\
\qquad[\sqrt{x}]\end{array}$ & $\begin{array}{l}\text { Prof. máxima } \\
{\left[\log _{10} x\right]}\end{array}$ & $\begin{array}{c}\text { Cantos rodados } \\
{[\sqrt{x}]}\end{array}$ & $\begin{array}{l}\text { Gravas } \\
{[\sqrt{x}]}\end{array}$ & Rep. lentos \\
\hline \multicolumn{8}{|l|}{$\overline{\text { Anchura }}$} \\
\hline$[\sqrt{x}]$ & 1 & $0.868 * *$ & 0.314 & $0.613^{* *}$ & -0.098 & 0.076 & 0.347 \\
\hline \multicolumn{8}{|l|}{ Superficie } \\
\hline$\left[\log _{10} x\right]$ & & 1 & 0.293 & $0.574 * *$ & -0.089 & 0.027 & 0.264 \\
\hline \multicolumn{8}{|l|}{ Prof. media } \\
\hline$[\sqrt{x}]$ & & & 1 & $0.786^{* *}$ & -0.151 & 0.019 & 0.319 \\
\hline \multicolumn{8}{|l|}{ Prof. máxima } \\
\hline$\left[\log _{10} x\right]$ & & & & 1 & -0.227 & 0.272 & 0.173 \\
\hline \multicolumn{8}{|l|}{ C. rodados } \\
\hline$[\sqrt{x}]$ & & & & & 1 & -0.391 & -0.372 \\
\hline \multicolumn{8}{|l|}{ Gravas } \\
\hline $\begin{array}{l}{[\sqrt{x}]} \\
\text { Rep. }\end{array}$ & & & & & & 1 & -0.290 \\
\hline lentos & & & & & & & 1 \\
\hline
\end{tabular}


sin embargo éstos no fueron seleccionados por la elevada colinealidad de sus variables (Tabla 4). Estos otros incorporaban las variables de superficie del hábitat y profundidad máxima, por lo que podemos considerar que estas variables también resultan significativas para interpretar las densidades y biomasas.

En cuanto a los modelos obtenidos el de densidad es mejor que el de biomasa, ya que éste último presenta un menor nivel de confianza. Este resultado concuerda con los modelos obtenidos por Jutila et al., (1999) en pequeños cauces de Finlandia para los juveniles de trucha común, en donde el modelo de densidad reflejaba mejor la variabilidad de datos (con un $r^{2}$ mayor) que el de biomasa.

En los dos modelos se han obtenido dos únicas variables independientes, anchura y cantos rodados. La anchura es la variable más importante en los dos modelos y presenta un efecto negativo, de forma que una disminución en la anchura del río se ha relacionado con un aumento en la densidad y biomasa de trucha común. Rosenfeld et al. (2002) encontraron esta misma relación con altas densidades de Oncorhynchus clarkii en pequeños ríos al Oeste de Canadá. Otros autores también han indicado que la anchura del río esta correlacionada con cambios en la composición de la comunidad acuática y en la distribución y abundancia de los peces (Vannote et al., 1980; Bozek \& Hubert, 1992).

A escala de un solo tramo, el aumento de la anchura para un caudal dado supone una lámina de agua menos profunda, lo que consideramos que es un factor crítico en estos ríos mediterráneos, donde el verano supone la época más

Tabla 5. Modelos de regresión múltiple para la densidad (ind./ha) $[\sqrt{x}]\left(r^{2}=0.450, p<0.006\right)$ y biomasa $(\mathrm{kg} / \mathrm{ha}) \mathrm{de}$ trucha común $[\sqrt{x}]\left(r^{2}=0.397, p<0.014\right)$. Multiple regression models for the density (ind./ha) $[\sqrt{x}]\left(r^{2}=0.450, p<0.006\right)$ and biomass $(\mathrm{kg} / \mathrm{ha})$ of the brown trout $[\sqrt{x}]\left(r^{2}=0.397\right.$, $p<0.014)$. Representatively of slow habitats.

\begin{tabular}{lccccc}
\hline & \multicolumn{3}{c}{ Densidad $[\sqrt{x}]$} & \multicolumn{3}{c}{ Biomasa $[\sqrt{x}]$} \\
\hline & $\begin{array}{c}\text { Coeficientes } \\
\text { de regresión }\end{array}$ & $p$ & $\begin{array}{c}\text { Coeficientes } \\
\text { de regresión }\end{array}$ & $p$ \\
\hline Constante & 6.527 & 0.002 & 4.303 & 0.009 \\
Anchura $[\sqrt{x}]$ & -2.392 & 0.008 & -1.410 & 0.049 \\
Cantos Rodados $[\sqrt{x}]$ & 0.272 & 0.070 & 0.276 & 0.030 \\
\hline
\end{tabular}

difícil para la supervivencia de las truchas. En este sentido, el aumento de anchura supone claramente un factor negativo para la supervivencia. Sin embargo, al tomar juntos 20 tramos de los cuatro ríos, estas circunstancia no se han reflejado en la correlación entre variables; por el contrario la correlación entre anchura y profundidad máxima fue positiva (Rho de Spearman $0.534, p=0.015)$. Según nuestras observaciones, consideramos que este resultado se debe a que los ríos con mayor caudal y con un cauce más desarrollado (Palancia y Villahermosa) presentan mayor anchura y profundidad máxima que los otros dos ríos. La aportación negativa de la anchura a los modelos de densidad y biomasa, en nuestra opinión, debería ser interpretada también en función de otras variables que no se han medido, principalmente la producción primaria y la disponibilidad de alimento que pueden ser los causantes de que la trucha común abunde más en los ríos más pequeños.

Por otro lado, se ha podido constatar la preferencia de la trucha común por el sustrato de cantos rodados (64-256 mm) como en otros estudios (Heggenes et al., 2002). Un resultado parecido obtuvieron Jutila et al., (1999) encontrando una relación positiva con el sustrato grueso en los ríos finlandeses, considerando un diámetro de la partícula entre 20 y $100 \mathrm{~mm}$ de diámetro. El área y distribución de las gravas para la freza tiene una importancia fundamental, ya que determina la productividad de un río y puede ser un factor limitante en la población de salmónidos de muchos ríos (Kondolf \& Wolman, 1993).

Una variable importante que no ha aparecido en los modelos es la profundidad máxima, por presentar un nivel de confianza inferior al $90 \%$ $(p>0.10)$. Consideramos que el hecho de que no haya sido seleccionada en el proceso de regresión paso a paso se debe a un enmascaramiento producido por la profundidad media, que está correlacionada con ella. Se ha demostrado que ambas variables están correlacionadas con la anchura en los tramos de estudio, por tanto podemos decir que la importancia de las tres variables ha quedado reconocida en el análisis, aunque solo una de ellas (anchura) ha aparecido finalmente en el modelo. Esperamos que la 
monitorización de estos tramos durante los años posteriores nos aporte una mayor información sobre el papel de cada una de estas variables en la dinámica poblacional de la trucha común.

La profundidad máxima junto con la velocidad media, caudal, tamaño del sustrato y refugio, están consensuadas en la literatura como variables explicativas de la distribución y abundancia de los salmónidos. El modo en que estas variables afectan a la distribución de los salmónidos ha sido demostrado en diversos estudios (Heggenes et al., 1999; Armstrong et al., 2003; LobónCerviá \& Rincón, 2004; Lobón-Cerviá \& Mortensen, 2005). Por ejemplo Jutila el al. (1999) demostraron que la profundidad media es el factor más importante en la selección del hábitat por la trucha común en ríos de Finlandia.

En los ríos mediterráneos, los sistemas acuáticos están profundamente influenciados por la torrencialidad de los caudales y las fuertes sequías (García de Jalón, 1992), por lo que consideramos que en nuestros ríos la profundidad máxima es una variable crítica. Así los lentos, pese a tener menor densidad media de recursos alimenticios, son más frecuentadas que los rápidos, pues suponen un refugio mucho mejor frente a las perturbaciones (Granado-Lorencio, 1996). Los lentos que tienen una adecuada profundidad, sirven de refugio a los salmónidos en periodos de severa sequía, ya que tanto la trucha común como la trucha arco iris buscan una adecuada temperatura y una buena concentración de oxigeno disuelto en las aguas, lo que las obliga a trasladarse hacia el fondo (Matthews \& Berg, 1997; Elliott, 2000). A pesar de su importancia en otros estudios, la representatividad de lentos, medida en segmentos de $300 \mathrm{~m}$, no aparece en los modelos por obtener un nivel de confianza inferior al $90 \%(p>0.10)$ y no se ha detectado su relación con otras variables, al menos entre las que cumplían la normalidad (Tabla 3).

Tampoco han sido incluidas las variables independientes que reflejan la calidad del agua y las características geográficas, por no cumplir con el principio de normalidad. En general, la calidad de las aguas es buena y sólo en algunos casos concretos se detectan pequeños incrementos en la conductividad de las aguas, debido a la mineralización de materia orgánica vertida. Destaca la dureza de las aguas que puede ser limitante por las consecuencias que las incrustaciones de carbonato cálcico tienen sobre los lechos de grava. Sin embargo las mediciones de este parámetro en todos los ríos resultaron ser muy homogéneas. Del mismo modo ocurre con las variables geográficas: la altitud, pendiente, distancia hasta el nacimiento y distancia hasta la desembocadura están dentro de un pequeño rango geográfico y es difícil obtener una adecuada distribución de los muestreos que refleje la variabilidad de estas variables a la escala de trabajo.

Por último cabe citar, como elemento limitante, que se presentan datos obtenidos únicamente en los meses de verano. A pesar de ello se ha podido demostrar la influencia de algunas variables del hábitat sobre las poblaciones de salmónidos en ríos ibéricos. También ha destacado el hecho de interpretar ambos modelos, densidad y biomasa, en función de las dos mismas variables, al menos a partir de los resultados obtenidos en un año de trabajo.

\section{AGRADECIMIENTOS}

Este estudio ha sido realizado por el Grupo de Evaluación de Impactos Ambientales del Dpto. de Ingeniería Hidráulica y Medio Ambiente (U. Politécnica de Valencia), con la financiación de la Consellería de Territorio y Vivienda de la Generalitat Valenciana. Agradecemos la labor realizada por Miguel Rodilla, Silvia Falco, Inma Romero, Remedios Martínez y Ángela Jaramillo en la determinación de los parámetros físicoquímicos del agua. A J. M. Soler Torro (Dpto. Estadística e Investigación Operativa) por su apoyo en la revisión de los análisis estadísticos. A Rafa Casas, Toni Pradillo y Roberto Coll por su apoyo en muestreos de campo.

\section{BIBLIOGRAFÍA}

ALMODÓVAR, A. \& G. G. NICOLA. 2004. Angling impact on conservation of Spanish stream- 
dwelling brown trout Salmo trutta. Fisheries Management and Ecology, 11: 173-182.

APHA. 1998. Standard methods for the examination of water and wastewater, 20th edition. American Public Health Association. American Water Works Association, Water Environment Federation. Washington. 769 pp.

ARMSTRONG, J. D., P. SKEMP, G. J. A. KENNEDY, M. LADLE \& N. J. MILNER. 2003. Habitat requirements of Atlantic salmon and brown trout in rivers and streams. Fisheries Research, 62: 143-170.

BOON, P. J., P. CALOW \& G. E. PETTS. (eds.). 1992. River conservation and Management. John Wiley \& Sons. 470 pp.

BOZEK, M. A. \& W. A. HUBERT. 1992. Segregation of resident trout in streams as predicted by three habitat dimensions. Can. J. Zool., 70: 886-890.

CARLE, F. L., \& M. R. STRUB. 1978. A new method for estimating population size from removal data. Biometrics, 34: 621-380.

COWX, I. G. 1983. Review of the methods for estimating fish population size from survey removal data. Fish. Mgmt., 14 (2): 67-82.

DOLLOFF, C. A., D. G. HANKIN \& G. H. REEVES. 1993. Basinwide estimation of habitat and fish populations in streams. U.S. Department of Agriculture. Forest Service. Southeastern Forest Experiment Station, $25 \mathrm{pp}$.

ELLIOTT, J. M. 2000. Pools as refugia for brown trout during two summer droughts: trout responses to thermal and oxygen stress. J. Fish Biol., 56: 938-948.

GARCÍA DE JALÓN, D. 1992. Dinámica de las poblaciones piscícolas en los ríos de montaña ibéricos. Ecología, 6: 281-296.

GONZÁLEZ DEL TÁNAGO, M. y D. GARCÍA DE JALÓN. 2001. Restauración de ríos y riberas. Ediciones Mundi-Prensa, $319 \mathrm{pp}$.

GRANADO-LORENCIO, C. 1996. Ecología de peces. Universidad de Sevilla, $353 \mathrm{pp}$.

HEGGENES, J., J. L. BAGLINIÈRE \& R. A. CUNJAK. 1999. Spatial niche variability for young Atlantic salmon (Salmo salar) and brown trout ( $S$, trutta) in heterogeneous streams. Ecology of Freshwater Fish, 8: 1-21.

HEGGENES, J., S. J. SALTVEIT, D. BIRD \& R. GREW. 2002. Static habitat partitioning and dynamic selection by sympatric young Atlantic salmon and brown trout in south-west England streams. J.Fish Biol., 60: 72-86.

JUTILA, E., A. AHVONEN \& M. LAAMANEN. 1999. Influence of environmental factors on the density and biomass of stocked brown trout, Salmo trutta L., parr in brooks affected by intensive forestry. Fisheries Management and Ecology, 6: 195-205.

KONDOLF, G. M. \& M. G. WOLMAN. 1993. The sizes of salmonid spawning gravels. Wat. Resour. Res., 29: 2275-2285.

LEOPOLD, L. B., M. G. WOLMAN \& J. P. MILLER. 1964. Fluvial Processes in Geomorphology. Freeman Co. San Francisco. 522 pp.

LOBÓN-CERVIÁ, J. \& E. MORTENSEN, E. 2005. Population size in stream-living juveniles of lake-migratory brown trout Salmo trutta L.: the importance of stream discharge and temperature. Ecology of Freshwater Fish, 14: 394-401.

LOBÓN-CERVIÁ, J. \& P. A. RINCÓN. 2004. Environmental determinants of recruitment and their influence on the population dynamics of streamliving brown trout Salmo trutta. Oikos 105: 641646.

MACHORDOM, A., J. SUÁREZ, A. ALMODÓVAR \& J. M. BAUTISTA. 2000. Mitochondrial haplotype variation and phylogeography of Iberian brown trout populations. Molecular Ecology, 9: 1325-1338.

MATTHEWS, K. R. \& BERG, N.H. 1997. Rainbow trout responses to water temperature and dissolved oxygen stress in two southern California stream pools. J. Fish Biol., 50: 50-67.

MEADOR, M. R., T. F. CUFFNEY \& M. E. GURTZ. 1993. Methods for sampling fish communities as part of the National Water-Quality Assessment Program. U.S. Geological Survey Open-File Report 93-104. Raleigh, North Carolina, USA, 40 pp.

ROSENFELD, J. S., S. MACDONALD, D. FOSTER, S. AMRHEIN, B. BALES, T. WILLIAMS, F. RACE \& T. LIVINGSTONE. 2002. Importance of Small Streams as Rearing Habitat for Coastal Cutthroat Trout. North American Journal of Fisheries Management, 22: 177-187.

STRAHLER, A. N. 1957. Quantitative analysis of watershed geomorphology. Transactions of the American Geophysical Union, 36: 913-920.

VANNOTE, R. L., MINSHALL, G. W., CUMMINS, K. W., SEDELL, J. R., \& CUSHING, C. E. 1980. The river continuum concept. Can. J. Fish. Aquat. Sci., 37: 130-137 
